${ }^{1}$ Centro de Educación Médica y Ciencias de la Salud (CEMCIS), Facultad de Medicina, Pontificia Universidad Católica de Chile. Santiago, Chile.

${ }^{2}$ Centro de Nutrición Molecular y Enfermedades Crónicas (CNMEC), Escuela de Medicina, Facultad de Medicina, Pontificia Universidad Católica de Chile. Santiago, Chile.

'Departamento de Nutrición, Diabetes y Metabolismo, Escuela de Medicina, Facultad de Medicina, Pontificia Universidad Católica de Chile. Santiago, Chile. aBioquímica.

bPsicóloga, Magister en Psicología Educacional. 'Psicóloga, Diplomada en Psicología Positiva. dIngeniera Civil, Magister en Bioprocesos. ePhD.

Trabajo financiado parcialmente por FONDECYT Proyecto No 1150340.

Los autores declaran no tener conflictos de interés.

Recibido el 15 de enero de 2019, aceptado el 24 de abril de 2019

Correspondencia a: Marcela Bitran CEMCIS, Facultad de Medicina, Pontificia Universidad Católica de

Chile, Diagonal Paraguay 362 , Santiago, Chile. mbitran@med.puc.cl

\section{Burnout en la formación de profesionales de la salud en Chile: Factores de protección y riesgo, y propuestas de abordaje desde la perspectiva de los educadores}

\author{
MARCELA BITRAN ${ }^{1,2, a, e}$, DENISSE ZÚÑIGA ${ }^{1, b}$, \\ NURIA PEDRALS ${ }^{2, c}$, GUADALUPE ECHEVERRÍA ${ }^{2,3, d,}$ \\ CLAUDIA VERGARA ${ }^{1}$, ATTILIO RIGOTTI ${ }^{2,3, e}$, KLAUS PUSCHEL ${ }^{1}$

\section{Burnout in students of health-care professions. Risk and protection factors}

Background: The burnout syndrome affects more than half of students and professionals involved in healthcare worldwide and is characterized by emotional exhaustion, depersonalization and a low perception of self-efficacy. Several studies indicate that when students are burnt-out, clinical work, professionalism and ethical behavior, as well as empathy, are negatively affected, while the risk of academic attrition, depression and suicidal ideation tend to increase. At a national level, recent information shows that one out of every two medical students suffer burnout at the beginning of the clinical cycle, a situation that does not improve after finishing undergraduate medical training. There is no consensus on which are the most appropriate strategies to face the problem of burnout in students and health-care professionals. Some studies indicate that the experience of medical and health educators may be key to the design of effective strategies to address this problem. Aim: To identify the burnout risk and protection factors of students at different medical schools. Material and Methods: In this study -in which 34 expert health educators from eight Chilean medical schools and other health-related schools participated-we used a qualitative methodology based on the appreciative inquiry to explore the key elements associated with the occurrence of burnout, identify protective and risk factors, as well as discuss possible effective interventions to prevent it. Results: There are personal, academic and contextual elements that act as protective or risk factors of burnout. In addition, the educators identified key elements to design organizational and curricular interventions to face the problem of burnout at a local level. Conclusions: Burnout is a serious problem in the formation of health care professionals. Teacher training aimed at promoting student'well-being must include the teaching of communication skills that consider both the generation gap and the profile of the professional medical schools intend to form.

(Rev Med Chile 2019; 147: 510-517)

Key words: Burnout, Professional; Burnout, Psychological; Chile; Protective Factors; Students, Medical. 
$\mathrm{E}$ burnout o síndrome de desgaste profesional afecta a más del $50 \%$ de los estudiantes y profesionales de la salud en el mundo ${ }^{1,2}$. Esta condición se describe como una respuesta al estrés crónico que se manifiesta como combinación de síntomas que incluyen el agotamiento emocional, la despersonalización o cinismo y la disminución del sentido de autoeficacia ${ }^{3,4}$. Estudios internacionales señalan que cuando los estudiantes sufren de burnout, la práctica clínica, el profesionalismo y la conducta ética ${ }^{5}$, así como la empatía ${ }^{6}$, se ven afectados negativamente, mientras que el riesgo de abandono de la carrera ${ }^{7}$, la depresión y la ideación suicida tienden a aumentar ${ }^{8}$.

La situación en Chile no es diferente a lo que ocurre en otros países más desarrollados. Un estudio multicéntrico de aprendizaje y bienestar en estudiantes de Medicina, en que participaron 1.395 estudiantes de once universidades chilenas, corroboró que uno de cada dos estudiantes sufre burnout al comenzar el ciclo clínico, situación que no mejora al terminar la carrera ${ }^{9}$. Más aun, este síndrome de agotamiento y despersonalización también afecta a una alta proporción de médicos residentes de nuestro país ${ }^{10,11}$.

En una primera etapa, el burnout tiende a pasar desapercibido pues puede coexistir con una alta motivación de los estudiantes por los estudios o el trabajo clínico y una conducta empática con el paciente ${ }^{12}$. Sin embargo, si el estudiante o profesional continua expuesto a altos niveles de estrés crónico, sin oportunidad de recuperarse, termina por perder su capacidad de cuidarse y de empatizar con el paciente y prestarle un cuidado de calidad ${ }^{12}$.

Afortunadamente, en los últimos años se ha configurado un cuerpo de evidencias acerca de cómo prevenir el burnout ${ }^{13,14}$. Los resultados de los meta-análisis más recientes coinciden en que, tanto las intervenciones enfocadas en los individuos como en los cambios organizacionales, pueden reducir el riesgo de burnout en médicos y estudiantes, particularmente el riesgo de agotamiento emocional ${ }^{14-16}$.

Recientemente, un colectivo de connotados educadores médicos, líderes académicos e investigadores expertos en burnout hizo un llamado urgente a la comunidad educativa internacional a actuar para mejorar el bienestar de los profesionales y jóvenes en formación ${ }^{17}$. Nuestro equipo docente ha hecho eco de este llamado ${ }^{18-20}$ a través de diversas actividades que incluyen el curso 'Pre- vención del burnout en la formación de profesionales de la salud' diseñado para docentes, de modo que éstos puedan actuar como agentes de cambio en sus respectivas instituciones.

\section{Metodología}

\section{Diseño}

El presente trabajo aplicó un enfoque cualitativo basado en indagación apreciativa, metodología orientada a identificar ("descubrimiento" o "indagación") aquellos aspectos que funcionan bien en un sistema u organización y que pueden resultar inspiradores para transformar una realidad determinada ${ }^{21,22}$. A partir de la identificación de estos aspectos claves, los participantes plantean un escenario ideal ("sueño") que les permitirá diseñar estrategias de intervención ("diseño") para cambiar el curso ("destino") del problema, mediante la ejecución del diseño propuesto. La indagación apreciativa se realiza en grupos pequeños de personas pertenecientes a un colectivo u organización que abordan aspectos críticos del problema o desafío mediante entrevistas rotativas, siguiendo una pauta de preguntas semiestructura$\mathrm{da}^{21}$. Decidimos aplicar la indagación apreciativa para iniciar el análisis del problema del burnout en la formación de profesionales en salud pues es una metodología que permite discurrir acciones preventivas, basadas en experiencias previas de éxito. Desde estos relatos será posible construir propuestas más ajustadas a la realidad de los participantes.

\section{Participantes, recolección y análisis de la información}

Este estudio se realizó en el contexto del curso 'Prevención del Burnout en la formación de profesionales de la salud' que se realizó en Santiago de Chile los días 8 y 9 de noviembre de 2018 en la Escuela de Medicina de la Pontificia Universidad Católica de Chile. Participaron 34 académicos con vasta experiencia en formación en salud de siete disciplinas diferentes (i.e., Medicina, Enfermería, Nutrición, Odontología, Kinesiología, Psicología y Educación) que representaban a ocho Escuelas de Medicina y de otras profesiones de la salud a nivel nacional. Los participantes otorgaron un consentimiento escrito a los docentes para la publicación y difusión de los resultados de su indagación. Esta 
investigación y la difusión de estos resultados cuentan con la aprobación del Comité de Ética Científico de la Facultad de Medicina UC.

Cada grupo estuvo conformado en promedio por 8 participantes (rango 5 a 9) y trabajó en dos sesiones de dos horas cada una. La primera sesión se dedicó a la fase de 'descubrimiento' y la segunda al 'sueño', es decir, la ideación de posibles programas de prevención de burnout. En la fase de 'descubrimiento', los participantes identificaron los factores protectores y de riesgo de burnout estudiantil así como las experiencias de éxito en el manejo de éste. Las reflexiones fueron recogidas en forma escrita por un participante en cada grupo. Posteriormente, cada grupo consolidó por escrito las contribuciones de sus integrantes y presentó al resto del curso un reporte oral de los resultados del proceso de indagación apreciativa.

Para la segunda sesión (diseño de posibles soluciones), los grupos trabajaron en torno a una pauta semiestructurada que contemplaba interrogantes acerca de las razones, características, alcance y evaluación de un posible programa de prevención del burnout estudiantil. El registro y reporte del trabajo grupal se realizó de manera semejante a la primera sesión.

Posteriormente, los registros escritos de ambas sesiones fueron analizados por dos investigadores y consolidados de modo de presentar la informa- ción de manera clara. Los resultados de la fase de 'descubrimiento' se reportan en este artículo como una matriz que consigna los factores de protección y de riesgo de burnout en el contexto de formación de pregrado en profesiones de la salud (Tablas $1 \mathrm{y}$ 2). Los resultados de la fase de 'diseño' se reportan en forma de texto, estructurado de acuerdo a las preguntas usadas en la indagación.

\section{Resultados}

Factores involucrados en el desarrollo de burnout durante la formación en profesiones de la salud en Chile.

\section{a) Antecedentes}

De acuerdo a la literatura, el burnout de los estudiantes en formación resulta de una conjunción de factores personales y relacionales, y de condiciones de la institución a que pertenecen ${ }^{23}$. A nivel personal y relacional, influyen la privación de sueño, la sobrecarga académica, el desbalance entre la vida personal y académica, los mecanismos evitativos de afrontamiento, crisis vitales durante la trayectoria académica, una baja percepción de autoeficacia, insuficiente autonomía para enfrentar responsabilidades profesionales crecientes, entre otras ${ }^{2,4,23}$. A nivel organizacional, inciden la

Tabla 1. Factores protectores de burnout en estudiantes chilenos de profesiones de la salud

\begin{tabular}{|c|c|c|}
\hline \multicolumn{3}{|c|}{ Factores de Protección de burnout } \\
\hline Individuales & Sociales & Institucionales \\
\hline $\begin{array}{l}\text { Resiliencia frente a experiencias } \\
\text { adversas durante la formación }\end{array}$ & Habilidades sociales & Buen clima educacional \\
\hline Asertividad en la comunicación & $\begin{array}{l}\text { Redes de apoyo amplias y } \\
\text { fuertes }\end{array}$ & $\begin{array}{l}\text { Enseñanza de profesionalismo en currículo } \\
\text { formal }\end{array}$ \\
\hline Valoración del autocuidado & $\begin{array}{l}\text { Participación en actividades } \\
\text { extracurriculares }\end{array}$ & Formación docente en bienestar y prevención \\
\hline \multicolumn{2}{|l|}{ Cultivo de estilos de vida saludables } & Adecuada proporción docente/alumno \\
\hline \multicolumn{2}{|l|}{ Desarrollo de la espiritualidad } & Sistema de evaluación del programa educacional \\
\hline \multirow[t]{5}{*}{ Pertenencia a la generación Y } & & Tamizaje precoz de estudiantes en burnout \\
\hline & & Oportunidades de coaching \\
\hline & & Disposición de residencias adecuadas \\
\hline & & Tiempos de traslado inter-campus breves \\
\hline & & Seguridad \\
\hline
\end{tabular}


Tabla 2. Factores de riesgo de burnout en estudiantes chilenos de profesiones de la salud

\begin{tabular}{|c|c|c|}
\hline \multicolumn{3}{|c|}{ Factores de Riesgo de burnout } \\
\hline Individuales & Sociales & Institucionales \\
\hline Alta autoexigencia y competitividad & Aislamiento social & Alta exigencia académica \\
\hline Baja tolerancia a la frustración & Vulnerabilidad social & Rigidez curricular \\
\hline Crisis vitales & & Evaluación centrada solo en objetivos cognitivos \\
\hline \multirow{5}{*}{$\begin{array}{l}\text { Insuficiente desarrollo de identidad } \\
\text { profesional }\end{array}$} & & Modelaje en torno al exitismo y/o autosuficiencia \\
\hline & & Alta carga asistencial \\
\hline & & $\begin{array}{l}\text { Falta de tiempo protegido para alimentarse y descansar } \\
\text { durante turnos y rotaciones }\end{array}$ \\
\hline & & Falta de recursos en el campo clínico \\
\hline & & Complejidad de los pacientes \\
\hline
\end{tabular}

falta de flexibilidad curricular, la escasa disponibilidad de tiempos protegidos para alimentación, descanso y autocuidado, la existencia de modelos profesionales que exaltan la autoexigencia y el sacrificio personal, el maltrato por parte de docentes y/o entre pares, la falta de apoyo (mentorías adecuadas, redes de tutores pares, derivación eficiente) y el aprendizaje en ambientes de salud 'estresados' ${ }^{\prime 2,23}$.

\section{b) Resultados de la indagación apreciativa}

Los participantes de este estudio identificaron un conjunto de factores de protección y riesgo para el desarrollo del burnout en sus propios contextos educativos, que están detallados en las Tablas 1 y 2.

Si bien muchos aspectos que contribuyen al riesgo de burnout son propios de la persona o sus circunstancias, existen aspectos institucionales que son susceptibles de modificar y que dependen de decisiones de las autoridades o de la interacción docente-estudiante. Dentro de los primeros, se mencionó la importancia de promover y facilitar el desarrollo de actividades extracurriculares que ayuden a los estudiantes a equilibrar la alta exigencia académica. En el ámbito relacional docente/ estudiante, una idea interesante fue la proposición de valorar positivamente algunos aspectos propios de la generación actual de estudiantes (Generación Y o Millennials) que favorecen el cultivo del propio bienestar ${ }^{24,25}$. Los asistentes reconocieron a los estudiantes actuales como un grupo humano que posee mayor conciencia de comunidad y con alta estima de la vida personal y familiar. Les caracteriza también un deseo de autonomía y una clara motivación a trabajar por propósitos propios $^{24,25}$. A los participantes del curso, les pareció un desafío socializar esta idea entre los docentes -especialmente de generaciones mayores- pues muchos de ellos suelen juzgar negativamente a sus estudiantes, especialmente su menor disposición al 'autosacrificio' en comparación con las generaciones anteriores ${ }^{24,25}$.

\section{Intervenciones para promover el bienestar de estudiantes de profesiones de la salud}

\section{a) Antecedentes}

Durante mucho tiempo, el enfoque predominante ha considerado el burnout como un problema individual indicativo de falta de resiliencia o vocación ${ }^{12}$. Ese análisis no incluía el posible rol en el malestar estudiantil de la institución educativa, su cultura institucional, exigencias y características. Sin embargo, a diferencia de la depresión y otras enfermedades mentales, el burnout es resultado de una interacción directa del individuo con su entorno laboral o académico. Por ende, es indispensable incluir en su análisis al ambiente y características institucionales, tanto para el diagnóstico del problema como para el diseño de soluciones $^{12,26}$.

Diversos metaanalisis de intervenciones -enfocadas tanto a nivel individual como institucionalindican que es posible reducir la tasa de burnout en 
estudiantes y profesionales de la salud ${ }^{14,15,27}$. Dentro de las intervenciones a nivel individual están, entre otras, talleres de manejo del estrés y autocuidado, resignificación de eventos adversos, comunicación efectiva y cultivo del bienestar mediante prácticas de atención plena o mindfulness ${ }^{27,28}$. A nivel organizacional, las acciones recomendadas son disminuir y racionalizar la carga de trabajo, aumentar la flexibilidad horaria, reestructurar los sistemas de turnos y formar a estudiantes $y$ docentes en habilidades de comunicación, trabajo en equipo y promoción del bienestar ${ }^{14,16,26}$.

\section{b) Resultados de la indagación apreciativa}

Los participantes del estudio elaboraron propuestas para la prevención del burnout y promoción del bienestar de sus estudiantes. A continuación se resumen las ideas centrales.

\section{¿Por qué desarrollar un programa de prevención de burnout?}

"Primero, porque nos cabe una responsabilidad ética y moral de formar profesionales con una salud y bienestar óptimos para el adecuado desempeño de su actividad profesional". El autocuidado ha sido reconocido como pilar fundamental del profesionalismo por entidades educacionales internacionales ${ }^{29,30}$ y fue consignado recientemente en la Declaración de Ginebra de la Organización Médica Mundial ${ }^{31}$. En opinión de los participantes, nuestro país debiera hacerse parte de esta iniciativa e incorporar transversalmente el autocuidado en los currículos de las diferentes carreras de la salud.

En segundo lugar, el burnout estudiantil tiene un gran impacto económico que gravita sobre los estudiantes, sus familias y las universidades. Este incluye los costos de atención en salud mental y también la pérdida económica asociada al abandono de la carrera o al aumento de la duración de los estudios. Por último, es necesario desarrollar programas de fomento del bienestar estudiantil porque el marco regulatorio de las carreras de la salud los hará exigibles próximamente para futuras acreditaciones de sus programas.

\section{¿Cuáles deben ser las características fundamenta- les del programa? \\ Para que un programa de prevención del burnout sea efectivo y sustentable, es preciso que esté inserto en una cultura de bienestar que com- prometa a la comunidad educacional en forma}

global y multiestamental: estudiantes, docentes, administrativos y autoridades.

\section{¿Cómo implementar el programa?}

Para implementar un programa de esta naturaleza, se debe contar con un comité mixto (estudiantes y académicos) para el bienestar, que dependa directamente del Decanato de la Facultad o Dirección de la Escuela correspondiente. Las labores de este comité deberían estar centradas en monitorización del bienestar y burnout, diseño e indicaciones de intervenciones a implementar, evaluación periódica y sistemática de las estrategias instauradas $y$, finalmente, gestar el cambio cultural institucional necesario para el éxito de estas intervenciones.

\section{¿A qué niveles se debe intervenir?}

A nivel curricular se plantearon cambios del currículo formal para incorporar el bienestar y autocuidado como parte de la enseñanza del profesionalismo, flexibilización de los programas de especialidad, formación en autocuidado y competencias transversales durante pre y postgrado e incorporación de mentorías por docentes y apoyo por pares.

A nivel institucional, surgieron diferentes ideas como generar conciencia del problema del burnout en la comunidad educativa, desarrollar espacios físicos amenos y amigables (en las escuelas mismas y en sus módulos docentes-asistenciales) para el descanso y satisfacción de necesidades básicas, promover actividades culturales y deportivas, generar encuentros de desarrollo docente con el objetivo de valorar el bienestar docente y estudiantil, capacitar en autocuidado y detección del burnout $y$ fortalecer iniciativas de acompañamiento entre pares e implementar estrategias individuales para su manejo y prevención.

\section{¿Cómo evaluar la efectividad del programa?}

El efecto de las intervenciones debe ser evaluado multidimensionalmente con indicadores cuantitativos y cualitativos. Se sugiere la incorporación de escalas como MBI (Maslach Burnout Inventory) ${ }^{32,33}$, MAAS (Mindful Attention Awareness Scale $)^{34,35}$, vitalidad emocional ${ }^{36,37}$ y MHC (Mental Health Continuum) ${ }^{38,39}$ que permitan un diagnóstico integral. Es necesario también monitorear las tasas de ausentismo académico, número de licencias médicas relacionadas con 
burnout y depresión, abandono y deserción de la carrera de pre o postgrado. Además de estos indicadores cuantitativos, es preciso realizar evaluaciones cualitativas de satisfacción y percepción de bienestar a nivel de estudiantes y docentes que permitan retroalimentar y modificar los programas. En general, el seguimiento debe considerar la recolección de datos provenientes de múltiples fuentes, incluyendo académicos preclínicos y clínicos, estudiantes, funcionarios administrativos, exalumnos e, idealmente, de los propios pacientes.

\section{Discusión}

Este estudio permitió conocer las distintas realidades que enfrentan los docentes responsables de la formación de profesionales de salud de nuestro país en cuanto al burnout de sus estudiantes.

La metodología de indagación apreciativa utilizada permitió descubrir las historias de éxito de los educadores en el manejo de este problema e identificar factores de protección y riesgo de burnout estudiantil en sus contextos educativos. Por otra parte, permitió a los participantes construir colaborativamente bases para diseñar programas de abordaje de la preocupante situación del burnout estudiantil.

Hubo consenso entre los participantes acerca de la responsabilidad que cabe a académicos y educadores como modelos y gestores del cambio para una mayor valoración del autocuidado y el cultivo del bienestar por parte de sus estudiantes. Más aun, los participantes expresaron su voluntad de diseñar e implementar intervenciones en sus propias instituciones. Es interesante destacar que entre los participantes del estudio había autoridades educacionales: directores de escuelas, jefes de programas de especialidad médica y coordinadores de salud estudiantil; todos ellos agentes claves en la toma de decisiones educativas.

Cualquier análisis del problema de burnout estudiantil no puede obviar el hecho que este es una manifestación de una disfunción de los sistemas de formación y prestación de salud. De hecho, los propios docentes y profesionales de la salud sufren niveles semejantes de burnout como resultado de la tensión docente-asistencial y la alta carga de responsabilidades en ambientes caracterizados por un estrés sostenido. No menos complejo es el tema de comunicación intergeneracional aludido por los participantes de este estudio. La propuesta de incluir una mirada apreciativa hacia las generaciones jóvenes resulta a la vez interesante y desafiante. Para generar conversaciones apreciativas y asertivas, es preciso contar con un repertorio comunicacional adecuado que permita a los docentes dar feedback del desempeño de sus estudiantes en materias relevantes a su formación, de manera y respetuosa y firme a la vez. Para ello, toda formación docente orientada a promover el bienestar debe incluir la enseñanza de habilidades comunicacionales que consideren tanto la brecha generacional como las características del profesional que queremos formar: un profesional de la salud capaz de brindar cuidados de excelencia a la vez que de cuidar de sí mismo, de sus estudiantes y de su equipo de trabajo.

Agradecimientos: Los docentes y autores de este artículo agradecemos a todos los participantes del curso por su generosidad al permitirnos compartir el producto de sus experiencias y reflexiones en torno al burnout y bienestar estudiantil. La experiencia de trabajar con académicos y autoridades de Universidad Católica del Norte, Universidad Finis Terrae, Universidad de los Andes, Universidad de Chile, Universidad del Desarrollo, Universidad Católica del Maule, Universidad de Concepción y Pontificia Universidad Católica de Chile reafirmó nuestra convicción como equipo docente y de investigación, que es una necesidad a nivel país incorporar programas de bienestar y autocuidado en la formación de profesionales de la salud.

Esperamos que esta experiencia estimule el desarrollo de una comunidad de educadores sensible e interesada en contribuir a la promoción del bienestar de nuestro estudiantes: una comunidad dispuesta a incluir 'el cuidado del cuidador' como objetivo clave en la formación de sus estudiantes, futuros profesionales a cuyo cuidado estará la salud de nuestra población.

\section{Referencias}

1. Dyrbye LN, West CP, Satele D, Boone S, Tan L, Sloan J, et al. Burnout among U.S. medical students, residents, and early career physicians relative to the general U.S. population. Acad Med 2014; 89 (3): 443-51.

2. Dyrbye L, Shanafelt T. A narrative review on burnout 
experienced by medical students and residents. Med Educ 2016; 50 (1):132-49.

3. Maslach C, Jackson SE. The measurement of experienced burnout. J Occup Behav 1981; 2: 99-113.

4. Maslach C, Leiter MP Understanding the burnout experience: Recent research and its implications for psychiatry. World Psychiatry 2016; 15 (2): 2-9.

5. Dyrbye LN, Massie FS, Eacker A, Harper W, Power D, Durning SJ, et al. Relationship between burnout and professional conduct and attitudes among US medical students. JAMA 2010; 304 (11): 1173-80.

6. Thomas NK. Resident Burnout. JAMA 2004; 292 (23): 2880-89.

7. Dyrbye LN, Thomas MR, Power DV, Durning S, Moutier C, Massie FS, Harper W, et al. Burnout and serious thoughts of dropping out of medical school: A multiinstitutional study. Acad Med 2010; 85: 94-102.

8. Dyrbye LN, Thomas MR, Massie FS, Power PD, Eacker A, Harper W, et al. Burnout and suicidal ideation among U.S. medical students. Ann Intern Med 2008; 149: 334-41.

9. Bitran M, Torres-Sahli M, Echeverría G, Zúñiga D, Pedrals N, Rigotti A. Dispositional mindfulness -a protective factor for burnout in medical students? Abstract Book 2018 Conference of the Association for Medical Education in Europe, Basel, Switzerland (p. 780).

10. Bitran M, González M, Nitsche MP, Zúñiga D, Riquelme A. Concern for residents' wellbeing, an issue at the Latin-American Conference on Residence Education 2017. Rev Med Chile 2017; 145 (10): 1330-5. DOI: 10.4067/ S0034-98872017001001330.

11. Díaz LA, Arab JP, Núñez C, Robles C, Bitran M, Nitsche $\mathrm{MP}$, et al. Burnout en médicos residentes de especialidades y subespecialidades: estudio de prevalencia y variables asociadas en un centro universitario' Ars Medica 2017; 42 (2). Doi:http://dx.doi.org/10.11565/arsmed. v42i2.541.

12. Maslach C, Leiter M. New insights into burnout and health care: Strategies for improving civility and alleviating burnout. Med Teach 2017; 39 (2): 160-3.

13. Haramati A, Cotton S, Padmore JS, Wald HS, Weissinger PA. Strategies to promote resilience, empathy and well-being in the health professions: Insights from the 2015 CENTILE Conference. Med Teach 2017; 39 (2): 118-9. Doi: 10.1080/0142159X.2017.1279278.

14. West CP, Dyrbye LN, Erwin PJ, Shanafelt TD. Interventions to prevent and reduce physician burnout: a systematic review and meta-analysis. Lancet 2016; 388 (10057): 2272-81. Doi: 10.1016/S01406736(16)31279-X. Epub 2016 Sep.

15. Panagioti M, Panagopoulou E, Bower P, Lewith G, Kon- topantelis E, Chew-Graham C, et al. Controlled Interventions to reduce burnout in physicians: A systematic review and meta-analysis. JAMA Intern Med 2017; 177 (2): 195-205. 10.1001/jamainternmed. 2016.7674.

16. West CP, Dyrbye LN, Shanafelt TD. Physician burnout: contributors, consequences and solutions. J Intern Med 2018; 283 (6): 516-29.

17. Ripp JA, Privitera MR, West C, Leiter R, Logio L, Shapiro J, et al. Well-being in graduate medical education: A call for action. Acad Med 2017; May 2 doi: 10.1097/ ACM.0000000000001735.

18. Pedrals N, Rigotti A, Bitran M. Aplicando psicología positiva en educación médica. Rev Med Chile 2011; 139: 941-9.

19. Pedrals N, Rigotti A, Bitran M. Focusing on Students' Talents and Strengths in Medical Education. Acad Med 2010; 85: 744-45.

20. Nitsche MP, Bitran M, González M. ¿Pueden los objetivos de un curso de mindfulness tributar a competencias del rol profesional CanMEDS relacionadas con el autocuidado? RIEM 2017; 6 (22): 131-2. https://doi. org/10.1016/j.riem.2017.01.128.

21. Cooperrider D, Sorensen PF Jr, Yeager TF, Whitney D, editors. Appreciative Inquiry: An Emerging Direction for Organization Development. Stipes Publishing LLC; Champaign, IL: 2001.

22. Bushe GR, 2000. Advances in Appreciative Inquiry as an Organizational Development Intervention. In D. L. Cooperrider, P. F. Sorenson, Jr., D. Whitney, and T. F. Yaeger (Eds.), Appreciative Inquiry: Rethinking Human Organization Towards a Positive Theory of Change. Stipes Publishing, Champaign, IL, pp. 113-21.

23. Dyrbye LN, Power DV, Massie F, Eacker A, Harper W, Thomas MR, et al. Factors associated with resilience to and recovery from burnout: A prospective, multiinstitutional study of US medical students. Med Educ 2010; 44 (10): 1016-26.

24. Waljee JF, Chopra V, Saint S. Mentoring Millennials. JAMA 2018; 319 (15): 1547-8.

25. Eckleberry-Hunt J, Tucciarone J. The challenges and opportunities of teaching "generation y". J Grad Med Educ 2011; 3 (4): 458-61. Doi: 10.4300/JGME-03-0415.

26. Shanafelt T, Trockel M, Ripp J, Murphy ML, Sandborg C, Bohman B. MD Building a Program on Well-Being: Key Design Considerations to Meet the Unique Needs of Each Organization. Acad Med 2018. Doi: 10.1097/ ACM.0000000000002415. [Epub ahead of print].

27. Daya $Z$ and Hearn J. Mindfulness interventions in medical education: A systematic review of their impact on medical student stress, depression, fatigue 
and burnout. Med Teach 2018; 40 (2); 146-53. Doi: 10.1080/0142159X.2017.1394999.

28. Epstein RM, Privitera MR. Doing something about physician burnout. Lancet 2016; 388 (10057): 2216-7.

29. Edwards S. Resident wellness and work/life balance in postgraduate medical education. Members of the FMEC PG consortium; 2011.

30. https://www.acgme.org/Meetings-and-Educational-Activities/Annual-Educational-Conference/Blog/Details/ ArticleID/6288/Physician-Well-Being-The-ACGME-and-Beyond

31. World Medical Association Declaration of Geneva. https://www.wma.net/policies-post/wma-declaration-of-geneva/.

32. Maslach C, Jackson SE, Leiter M. The Maslach Burnout Inventory (3rd ed.). Palo Alto, CA: Consulting Psychologists Press. 1996.

33. Gill-Monte P. Factorial validity of the Maslach Burnout Inventory (MBI-HSS) among Spanish professionals. Rev Saúde Pública 2005; 39 (1): 1-8.

34. Brown KW, Ryan RM. The benefits of being present:
Mindfulness and its role in psychological well-being. J Pers Soc Psychol 2003; 84: 822-48.

35. Soler J, Tejedor R, Feliu-Soler A, Pascual J, Cebolla A, Soriano J, et al. Psychometric properties of Spanish version of Mindful Attention Awareness Scale (MAAS). Actas Esp Psiquiatr 2012; 40 (1): 19-26.

36. Ryan RM, Frederick C. On energy, personality and health: Subjective vitality as a dynamic reflection of well-being. J Pers1997; 65: 529-65. https://doi.org/10.1111/j.1467-6494.1997.tb00326.x.

37. Castillo I, Tomas I and Balaguer I. The Spanish-version of the subjective vitality scale: psychometric properties and evidence of validity. Span J Psychol 2017; 20 (e26): 1-8. Doi: $10.1017 /$ sjp.2017.22

38. Keyes C. The mental health continuum: From languishing to flourishing in life. JHSB 2010; 43: 207-22.

39. Echeverría G, Torres M, Pedrals N, Padilla O, Rigotti A, Bitran M. Validation of a Spanish version of the Mental Health Continuum-Short Form Questionnaire Psicothema 2017; 29 (1): 96-102. Doi: 10.7334/psicothema2016.3. 